\title{
BILATERAL MACULAR COLOBOMA AND MULTIPLE SYSTEMIC ASSOCIATIONS: AN INTERESTING CASE STUDY
}

Harapanahalli Venkatesh Soumya ${ }^{1}$, Sastry Purushottam² ${ }^{2}$, Sundaramurthy Harsha ${ }^{3}$, Channanayak Chandrashekhar ${ }^{4}$, Bechavalli Shanthappa Mahesh ${ }^{5}$

\section{HOW TO CITE THIS ARTICLE:}

Harapanahalli Venkatesh Soumya, Sastry Purushottam, Sundaramurthy Harsha, Channanayak Chandrashekhar, Bechavalli Shanthappa Mahesh. "Bilateral Macular Coloboma and Multiple Systemic Associations: An Interesting Case Study". Journal of Evolution of Medical and Dental Sciences 2014; Vol. 3, Issue 29, July 21; Page: 8067-8071, DOI: 10.14260/jemds/2014/3013

ABSTRACT: Macular colobomata refer to circumscribed, excavated defects in the choroid and retina at the macular area associated with decreased vision. Previous reports have speculated various causes for its occurrence. Familial cases of macular coloboma have roused the possibility of genetic etiology. Macular colobomas associated with systemic and other ocular abnormalities have been attributed to be either embryological maldevelopment or inflammatory etiology. Macular colobomata associated with skeletal abnormalities have been reported frequently. We report a 14-year old boy with an unusual presentation of extensive bilateral macular coloboma with planovalgus feet dynamics and left foot drop due to anterior horn cell disease.

KEYWORDS: Macular colobomata, bilateral macular coloboma, ocular coloboma.

MESHTERMS: Coloboma, Macula Lutea/abnormalities.

INTRODUCTION: Macular colobomata refer to circumscribed, excavated defects in the choroid and retina at the macular area associated with decreased vision. Previous reports have speculated various causes for its occurrence. Familial cases of macular coloboma have roused the possibility of genetic aetiology. Macular colobomas associated with systemic and other ocular abnormalities have been attributed to be either embryological maldevelopment or inflammatory aetiology. Macular colobomata associated with skeletal abnormalities have been reported frequently.

We report a 14-year old boy with an unusual presentation of extensive bilateral macular coloboma with planovalgus feet dynamics and left foot drop due to anterior horn cell disease.

CASE REPORT: A 14-year old boy presented to the outpatient department of ophthalmology with history of diminution of vision in both eyes and squinting of left eye since birth. He had difficulty in standing, walking and frequent falls since birth; which gradually progressed. The boy was nursed in neonatal intensive care unit for the first 15 days following birth - probably indicative of a premature birth. His developmental milestones were delayed. No history of consanguinity or no similar complaints with the parents or siblings.

On ophthalmic examination, his best corrected visual acuity was 20/120 in right eye and $20 / 200$ in left eye with eccentric fixation. His paramacular refraction was variable but revealed a low myopic astigmatic error. His colour vision was normal in right eye and abnormal in the left. Anterior segment examination including pupils was normal. He had an alternating exotropia of 15 degrees and pendular nystagmus in horizontal gaze. Ocular movements were normal.

His dilated fundus examination revealed bilateral, circumscribed, excavated, macula involving coloboma, in the right eye measuring about $4 \times 4$ disc diameters and in the left about $5 \times 5$ disc 
diameters. These discrete well defined deeply excavated areas were surrounded by pigmentation at places with ectatic sclera at the base.

A large choroidal vessel was visible at the base with pigment clumps around it. Triangular area of scarring was present adjacent to the nasal margin of coloboma in right eye (Figure 1). In the left eye, thin atrophic neurosensory retina with retinal vessels covered the coloboma except in the central area where sclera was ectatic. Inferonasal to the coloboma, a localized area of retinal detachment could be made out. (Figure 1). Both the discs appear tilted with areas of peripapillary atrophy. Retinal vessels appeared slightly tortuous adjacent to disc. Peripheral retina showed white without pressure areas.

Skeletal examination revealed flat chest, planovalgus feet dynamics with high stepping gait. Both the feet showed overriding of $3^{\text {rd }}$ toe with increased space between great and second toe (Figure 2). His neurological examination revealed wasting and weakness of distal muscles, left foot drop suggestive of anterior horn cell disease. He had behavioural problems in the form of laughing and talking to one's own self, easy irritability and poor scholastic performance. Examination of other systems was normal.

A systematic evaluation including complete blood count, Erythrocyte sedimentation rate, and renal function tests, chest x-ray were normal. His serology for toxoplasmosis, syphilis, cytomegalovirus, human immunodeficiency virus was negative. Radiographs of the feet revealed widened web space between great and second toe. (Figure 2). Fundus fluorescein angiography was not done since the boy was not co-operative. Optical coherence tomography revealed absence of retinal pigment epithelium and choroid. (Figure3).

DISCUSSION: Ocular coloboma is defined as an absence of ocular tissue due to failure of a part of fetal fissure to close ${ }^{1}$. Macular coloboma is grouped under atypical coloboma since it involves those areas of eye, which do not arise from embryonic fissure closure. ${ }^{2}$

Ida Mann classified macular coloboma into three types, pigmented, non-pigmented and macular coloboma associated with abnormal vessels. ${ }^{3}$ The present case may probably be grouped under type 2 coloboma.

Cases of bilateral and unilateral macular coloboma have been reported in the literature with other ocular and systemic associations.

Freedman et al have described bilateral macular coloboma with keratoconus and retinitis pigmentosa. ${ }^{4}$

Chen et al described a case of bilateral macular coloboma with aggregation of pigment clumps and speculated that it is developmental abnormality in nature. ${ }^{5}$

Macular colobomata with skeletal abnormalities have been reported by four different authors previously.

Sorsby in his summary of macular coloboma has described a pedigree of a mother and five of her eight children who all had bilateral macular coloboma, nail abnormalities and apical dystrophy of hands and feet. ${ }^{6}$

Phillips and Griffith reported a brother and sister who had bilateral macular coloboma, cleft palate, hallux valgus and other abnormalities. Since two out of four siblings were affected, they suggested an autosomal recessive mode of inheritance, considered inflammation to be unlikely and 
speculated the cause to be defect in the development of cone-bearing area of retina and/or related choroid or sclera. ${ }^{7}$

Smith RD et al reported association of severe short-limbed dwarfism and macular coloboma with histology resembling diastrophic dysplasia and postulated it to be a syndrome of as yet unknown cause. ${ }^{8}$

Alsing A et al reported bilateral atypical coloboma, familial juvenile nephronophthisis and skeletal dysplasia of upper limbs with bilateral radio humeral fusion in two sisters and proposed it to be a new oculo-reno-skeletal syndrome. ${ }^{9}$

JooYoun $\mathrm{Oh}$ et al have explained optical coherence tomographic findings in a macular coloboma. They showed a crater-like depression accompanying atrophic neuro-sensory retina and an absence of retinal pigment epithelium and choroid. ${ }^{10}$

The present case had some findings similar to Sorsby, but nothing to suggest consanguinity between two families. Hands were normal and only abnormalities of feet were noted. This patient had planovalgus and no hallux valgus.

Additional findings were an overriding $3^{\text {rd }}$ toe in both feet, increased web space between toes. He also had neurological and behavioural problems. He was tall for his age and did not exhibit shortlimbed dwarfism as in Smith RD or skeletal dysplasia as in Alsing A case studies.

Optical coherence tomographic findings in our case resembled that of JooYoun Oh et al, in showing absence of retinal pigment epithelium and choroid with thin atrophic neurosensory retina.

To conclude, this patient had macular coloboma and an adjacent area of scarring which may postulate an intrauterine inflammation as the cause, albeit serology being negative.

Sporadic occurrence of macular coloboma with no abnormalities in parents or siblings may grossly rule out a genetic element, but the associated skeletal, neurological and behavioural abnormalities may hint towards a syndrome of unknown aetiology.

ACKNOWLEDGEMENT: We are grateful to Dr Veena C N, retina consultant, for her kind co-operation in optical coherence tomography.

\section{REFERENCES:}

1. Duke - Elder S, Cook C. congenital deformities, In: Duke- Elder S, Cook C, eds System of Ophthalmology. St Louis: Mosby Company, 1963; Vol 6 Chap 14.

2. Pagon RA. Ocular coloboma. Surv Ophthalmol 1981; 25: 223-36.

3. Mann IC. On certain abnormal conditions of the macular region usually classed as coloboma. Br J Ophthalmol 1927; 11: 99-116.

4. Freedman J, Gombos GM. Bilateral macular coloboma, keratoconus and retinitis pigmentosa. Ann Ophthalmol 1971; 3 (6): 664-5.

5. MS Chen, $\mathrm{CH}$ Yang, JS Huang. Bilateral macular coloboma and pigmented paravenous retinochoroidal atrophy. Br J Ophthalmol 1992; 76: 250-251.

6. Sorsby A. Congenital coloboma of the macula: together with an account of the familial occurrence of bilateral macular coloboma in association with apical dystrophy of hands and feet. Br J Ophthalmol 1935; 19: 65-90.

7. Phillips CI, Griffiths DL. Macular coloboma and skeletal abnormality. Br J Ophthalmol 1969; 53: 346-9. 


\section{CASE REPORT}

8. Smith RD, Fineman RM, Silience DO et al. Congenital macular colobomas and short-limb skeletal dysplasia. Am J Med Genet 1980; 5 (4): 365-71.

9. Alsing A, Christensen C. Atypical macular coloboma (dysplasia) associated with familial juvenile nephronophthisis and skeletal abnormality. Ophthalmic Paediatr Genet 1988; 9 (3): 149-55.

10. Oh JY, Yu YS, Hwang JM et al. Optical Coherence Tomographic finding in a case of macular coloboma. Kor J Ophthalmol 2007; 21 (3): 175-177.

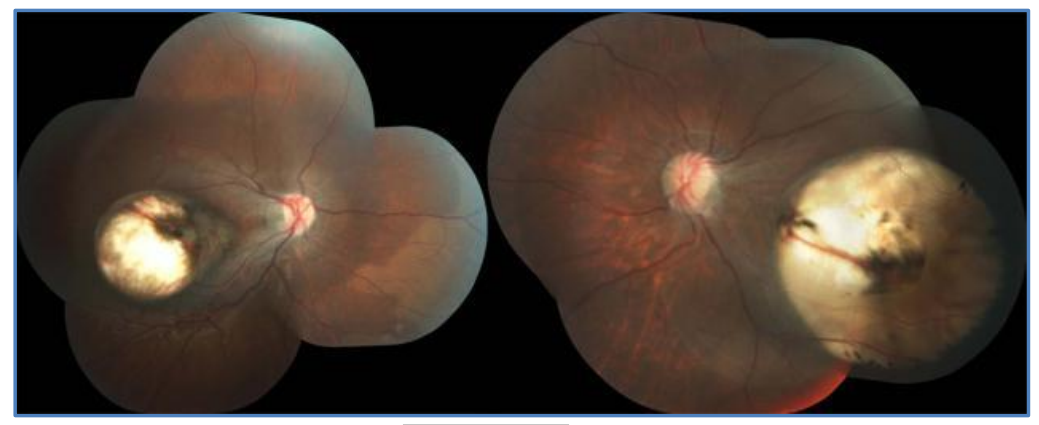

Fig: 1

Figure 1: Bilateral macular coloboma. The right eye shows area of scarring nasal to coloboma. The left eye shows thin retina covering part of macular coloboma. Feet showing widely spaced $1^{\text {st }}$ and $2^{\text {nd }}$ toe, $2^{\text {nd }}$ toe being longer than $1^{\text {st. }}$. All the other toe show varying degrees of flexion.

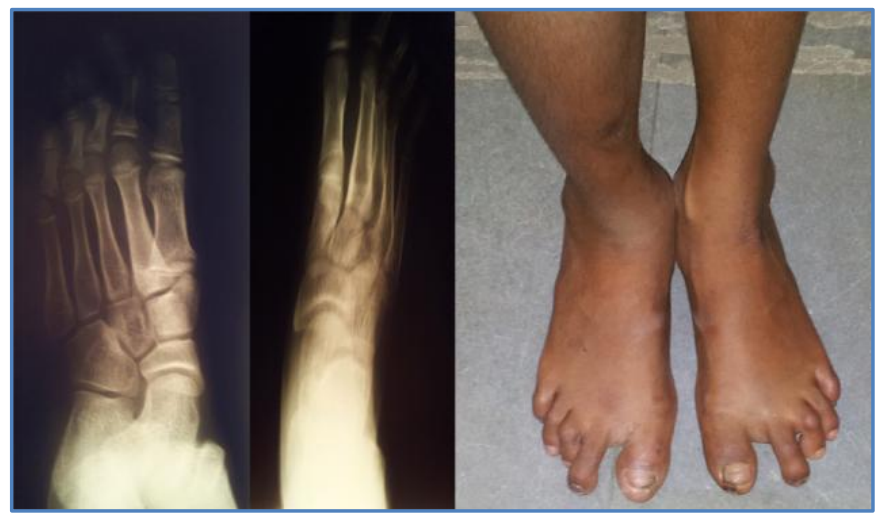

Fig: 2

Figure 2: Radiographs of feet showing increased space between phalanges with terminal

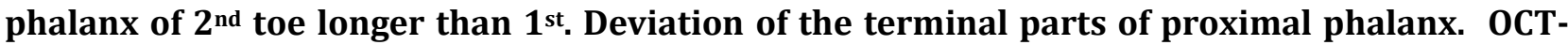
right eye showing area of scarring represented by high reflectivity and absence of retinal pigment epithelium and choroid. 


\section{CASE REPORT}

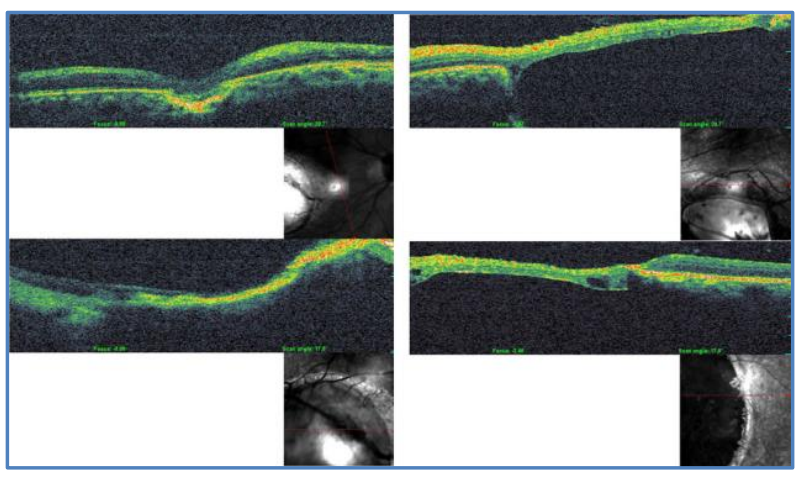

Fig: 3

Figure 3: OCT- left eye showing thin neurosensory retina and abrupt termination of choroid and retina at the interface.

\section{AUTHORS:}

1. Harapanahalli Venkatesh Soumya

2. Sastry Purushottam

3. Sundaramurthy Harsha

4. Channanayak Chandrashekhar

5. Bechavalli Shanthappa Mahesh

\section{PARTICULARS OF CONTRIBUTORS:}

1. Assistant Professor, Department of Ophthalmology, JSS Medical College Hospital, Mysore, Karnataka, India.

2. Professor, Department of Orthopaedics, JSS Medical College Hospital, Mysore, Karnataka, India.

3. Professor, Department of Neurology, JSS Medical College Hospital, Mysore, Karnataka, India.

4. Associate Professor, Department of Pediatrics, JSS Medical College Hospital, Mysore, Karnataka, India.
5. Professor, Department of Ophthalmology, JSS Medical College Hospital, Mysore, Karnataka, India.

\section{NAME ADDRESS EMAIL ID OF THE CORRESPONDING AUTHOR:}

Soumya Harapanahalli Venkatesh, Assistant Professor, Department of Ophthalmology, JSS Medical College Hospital, Ramanuja Road, Agrahara, Mysore-570004,

Karnataka, India.

Email: drhvsoumya@yahoo.co.in

Date of Submission: 04/07/2014. Date of Peer Review: 05/07/2014. Date of Acceptance: 12/07/2014. Date of Publishing: 17/07/2014. 